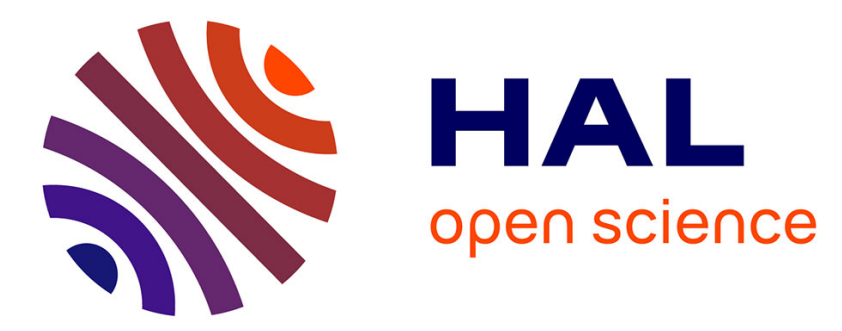

\title{
Dynamic response of a vaporizing spray to pressure oscillations: Approximate analytical solutions
}

Kwassi Anani, Roger Prud'Homme, Mahouton Norbert Hounkonnou

\section{To cite this version:}

Kwassi Anani, Roger Prud'Homme, Mahouton Norbert Hounkonnou. Dynamic response of a vaporizing spray to pressure oscillations: Approximate analytical solutions. Combustion and Flame, 2018, 193, pp.295-305. 10.1016/j.combustflame.2018.03.015 . hal-02013506

\section{HAL Id: hal-02013506 https://hal.sorbonne-universite.fr/hal-02013506}

Submitted on 11 Feb 2019

HAL is a multi-disciplinary open access archive for the deposit and dissemination of scientific research documents, whether they are published or not. The documents may come from teaching and research institutions in France or abroad, or from public or private research centers.
L'archive ouverte pluridisciplinaire HAL, est destinée au dépôt et à la diffusion de documents scientifiques de niveau recherche, publiés ou non, émanant des établissements d'enseignement et de recherche français ou étrangers, des laboratoires publics ou privés. 


\title{
Dynamic Response of a Vaporizing Spray to Pressure Oscillations: Approximate Analytical Solutions
}

\author{
Kwassi Anani $^{1,2, *}$, Roger Prud'homme ${ }^{3}$ and Mahouton Norbert Hounkonnou ${ }^{4}$
}

${ }^{1}$ Doctor, Université de Lomé,

Faculté des Sciences,

Département de Mathématiques,

02 BP 1515 Lomé, Togo

E-mail: kanani@univ-lome.tg

Tel.: (+228) 22255094

Fax: (+228) 22218595

${ }^{2}$ Université de Lomé,

Faculty of Sciences,

Laboratory of Renewable Energies, UNESCO Chair,

02 BP 1515, Lomé, Togo

${ }^{3}$ Emeritus Research Director, Jean Le Rond d'Alembert Institute, UMR 7190 - Pierre et Marie Curie University / Centre National de la Recherche Scientifique - Box 162 - 4 place Jussieu - 75252 Paris Cedex 05

E-mail: roger.prud_homme@,courriel.upmc.fr

${ }^{4}$ Professor, University of Abomey-Calavi, International Chair in Mathematical Physics and Applications (ICMPA-UNESCO Chair) 072 B.P. 050 Cotonou, Republic of Benin E-mail: norbert.hounkonnou@cipma.uac.bj

\section{Full-length article}

*Corresponding author: kanani@,univ-lome.tg 


\section{Abstract}

In this work, we study thermal conduction and convection combined effects on frequency response to pressure oscillations of a spray of repetitively injected drops in a combustion chamber. The theoretical model is based on Heidmann analogy of the so called "mean droplet" which is a single spherical vaporizing droplet with constant average radius, given that this droplet is continually fed at a stationary flow rate. The feeding comes from a source point placed at the mean spherical droplet centre in such a way that the injection process can be assumed to be isothermal (isothermal feeding regime) or adiabatic (adiabatic feeding regime). Drawing upon the linear decomposition of the energy conservation equation, approximate analytical solutions for the perturbed temperature field inside the droplet are obtained from some derived double confluent Heun equations. Frequency response factor of the evaporating mass is then evaluated on the basis of the Rayleigh criterion by means of the linearized equations of the gas phase. Compared to the results obtained for the previous pure conduction model of the same "mean droplet", frequency response factor curves seem to be similar with reference to each feeding regime. Moreover, due to the radial thermal convection effect introduced in the present work, a frequency response factor curve with the same characteristic times ratio may exhibit a relatively larger frequency range for the instability domain. Data are found to be correlated in terms of period of pressure oscillations, vaporization characteristics times and of fuel thermodynamic coefficients. In the isothermal feeding regime in particular, due to some possible values that can be taken by a certain thermodynamic coefficient, high and non-linear frequency responses may appear in the system.

Keywords: mean spherical droplet; frequency response factor; adiabatic or isothermal feeding regime; double confluent Heun equation; approximate analytical solution 


\section{Introduction}

Combustion instabilities are still being extensively studied nowadays though a great amount of theoretical, numerical, experimental works were devoted to their modelling and control during the past decades. Indeed, flow oscillations induced by external forcing or self-excited combustion instabilities occur in all types of combustion system (power generation engines, aeronautic engines and aerospace engines). A stable combustion process can be generally characterized by small amplitude pressure fluctuations as for example, less than about $5 \%$ of the mean chamber pressure for certain combustors [1]. But, interactions between the fuel vaporization, heat release and oscillatory flow field in combustion systems can cause departure from stable operating conditions. Further, fuel and operational flexibility requirements of new type of combustor architectures involve more complex and less predictable vaporization and acoustic couplings. In particular, the mechanism of spray combustion instabilities is hardly understood. In one hand, coupling between small acoustic oscillations in pressure and mass release at certain frequency levels may eventually lead to engine failure and other catastrophic consequences. In the other hand, particularly newer blends of liquid fuels can be engineered to undergo preferential instabilities leading to homogeneous combustion with higher efficiency [2]. In most diffusion flame models, many processes were pointed out as being responsible for triggering or for controlling high and nonlinear pressure oscillations in stable combustion chamber: combustor geometry [1], fuel type and composition [3], injection and atomization mechanisms [4], droplets vaporization characteristic times and other boundary conditions [3, 5-7], fuel/air mixing [8], ...Those studies are usually validated by experiments on particular practical fuels.

It appears from the above discussions that theoretical models are needed, which provide more details on the sequence of some mechanisms leading to high and nonlinear pressure 
oscillations occurrence in a stable combustion chamber. Considering two different fuel injection regimes, the present subcritical diffusion flame model includes both effects of process characteristics times and of fuel thermodynamic coefficients on the frequency response of a vaporizing spray. The evaluation of the frequency response of the vaporization process to pressure oscillations is mostly based on single vaporizing droplet models $[3,5,9]$. In other theoretical studies, as generally practised in most numerical simulations, the dynamic behaviour of spray vaporization is taken as a statistical consequence of the vaporization characteristics of each individual droplet in the array. Examples of these spray models are those of Harrje and Reardon [6] and of Delplanque and Sirignano [4]. Tong and Sirignano [10] also have examined the response of vaporizing droplets to oscillating ambient pressure and velocity conditions. The oscillatory rate of vaporization of an array of repetitively injected droplets in the combustion chamber is obtained from summation of individual droplet histories. In the same context, let us cite DiCicco and Buckmaster [11], Dubois et al. [12] and Sirignano et al. [7]. In all the above-mentioned studies, the actual changing volume due to the vaporization of the injected droplets has been taken into account.

During the 1960s, in order to study the instabilities generated or amplified by evaporation, Heidmann and Wieber the first replaced in their models, the spray of repetitively injected drops in the combustion chamber by a mean evaporating motionless spherical droplet [13, 14]. This mean droplet which has a constant average radius is continuously supplied with a stationary flow rate of the same fluid. The mean diameter of this average drop is considered to be invariant, as the supplied liquid compensates the evaporation. This evaporating droplet represents a mean spherical droplet with constant volume, at a specific location in the combustion chamber, and is supposed to summarize the frequency response of individual drops in the spray. The acoustic oscillations really affect the vaporizing droplet acceleration 
as well as its heat and mass transfer processes by giving the droplet three-dimensional velocity components and also causing perturbations within the droplet temperature and evaporation rate. But, Heidmann and Wieber's numerical evaluation of the frequency response showed that the velocity difference contributions to the vaporization rate at high and low values of the ambient oscillating pressure were nearly equal and thus cancelled effects with regard to response factor evaluation. Therefore, for an approximation the velocity difference effects on vaporization rate were ignored. In the present paper, adopting this hypothesis, we consider, the case of a velocity-stabilized mean spherical droplet representing a spray of repetitively injected droplets in the combustion chamber. This evaporating droplet with constant average radius is continuously fed by a steady flow.

The main simplified assumption of Heidmann and Wieber in their model described above is that an infinite thermal diffusivity of the liquid phase is supposed; therefore the mean spherical droplet has a uniform temperature whatever the feeding process adopted. This classical model was reviewed and substantially refined in 2009 by Prud'homme et al. [15]. Their analysis, which takes into account a finite thermal diffusivity of the liquid, was equally based on certain other simplifying assumptions. Namely, it was assumed that the supplied spherical droplet centre remains adiabatic (zero temperature gradient) and that the radial convection effect in the energy equation due to the feeding process at the centre of the droplet is neglected. Explicit analytical expression was then derived for the droplet mass transfer function in that pure conduction case and the results were discussed. With an aim of extending the analytical expressions of the perturbed evaporating rate and to the temperature field in a spherical symmetry configuration, Anani and Prud'homme (2016) have recently studied the pure thermal conduction effect on the perturbed mean spherical droplet and have pointed out the specificity of the isothermal injection regime [16]. The results derived from 
this case were then compared to that of the adiabatic feeding case. It was shown that, high and non-linear frequency responses may appear in the process when taking a particular thermodynamic coefficient value inferior but closer to that of a specific characteristic times ratio. Nevertheless this recent study remains all the same based on the simplifying assumption of a negligible radial thermal convection effect inside the liquid-phase. Apart from the pure thermal conduction model cases, no analytical solution has been found and any asymptotical study has been performed for the mean spherical droplet model with a finite thermal diffusivity.

The present paper aims at contributing to that sequence of linear analysis of harmonic instability mechanisms in combustion chambers by analytical approaches. This new model takes into account thermal conduction and convection combined effects on the dynamic response of the mean spherical droplet with a finite thermal diffusivity. In the analysis, double confluent Heun equations [17] will be derived from the linearized energy conservation equation of the liquid phase and approximate analytical solutions of the perturbed temperature field inside the mean spherical droplet will be obtained for both feeding regimes. Based on Rayleigh criterion [18], a frequency response of the vaporization process to small harmonic oscillations in pressure will be evaluated for both feeding regimes. Variations in the frequency response factor curves with the vaporization characteristic times ratio will also be presented. Results comparisons will then be made between the two different feeding regimes as they represent each other a specific boundary condition controlling the whole injection process. The mass response factor of the mean droplet model with thermal convection effect will be equally compared to that of the pure conduction model and results will be analysed. 


\section{Description of the unperturbed state}

Today's most theoretical studies of combustion instability in liquid-fuelled engines are based on numerical models and simulations of single vaporizing droplet dynamic response to ambient pressure and/or velocity perturbations (e.g., [3, 5]). But, even while assuming certain simplifying assumptions, analytical approaches that include more complex aspects of the problem are needed to get a better understanding of the mechanism. Those approaches may then serve to improve the development of numerical codes as for instance Computational Fluid Dynamic (CFD) codes (see [19]). One of those analytical approaches can stem from Heidmann analogy of a spherical vaporizing droplet of constant volume.

\subsection{General assumptions}

As mentioned in the introduction, the computation of the dynamic response of the vaporization process to pressure oscillations is mostly based on classical spray vaporization models. Existing evaporation models which are applicable to modern many-droplet calculations as usually performed in CFD codes were reviewed by Miller et al. (1998) in [20]. Therein, eight sub-models were compared and efforts were made to subject theoretical predictions of each model to experimental scrutiny. Results show that the model based on non-equilibrium Langmuir-Knudsen evaporation law formulation with an infinite liquid conductivity should be used for general gas-liquid flow calculations. Thereby, this model is shown to require less computational effort than the remaining models and to provide more accurate predictions. The Abramzon and Sirignano model [21] is also shown to provide improved agreement with experimental results, compared especially to the classical rapid mixing model. But, their model may be costly for many-droplet simulations. However, in the present study, the vaporizing spray is modelled by a single mean spherical droplet. As a consequence, the gas-phase sub-model equations used in the present paper are those derived by Prud'homme et al. [15] from the Abramzon and Sirignano model. These equations are 
briefly recalled in subsection 2.3 for a free vaporizing droplet at its unperturbed or stabilized state. For such a free vaporizing droplet, that is a single injected droplet during its course of evaporation, this state corresponds to a state of rest in an infinite atmosphere and is characterized by $[15,16]$ : constant average evaporation rate, uniform temperature of saturated vapour at the transient droplet surface, and equal velocities of the ambient gas flow and of the droplet. Below, these assumptions will be discussed in connection to the stabilized mean spherical droplet. Concerning the liquid phase sub-models usually used in CFD codes, five different groups classed in ascending complexity order were reported by Sazhin et al. (2006) in their comparative analysis review [22]. Among the classes, the present description of the supplied droplet at the stabilized state may be relevant to class four, as both finite liquid thermal conductivity and liquid re-circulation (restrained here to the radial convection) are considered for the droplet. Indeed, contrary to the classical model of Heidmann, our present model does not assume that the mean droplet reaches a steady and uniform temperature in the interior even when that droplet is in its stabilized state. From now on, all primed quantities will denote perturbed quantities (i.e. $\left.x^{\prime}=(x-\bar{x}) / \bar{x}\right)$ whereas all barred quantities will indicate mean values corresponding to the stabilized state.

In the physical model, the feeding is supposed to be done at the centre of the mean droplet and the liquid circulation within the droplet is distributed throughout in order to maintain its spherical symmetry configuration. During the injection, the centre of the droplet is assumed to be adiabatic (zero temperature gradient) or isothermal (imposed constant temperature). These two thermal regimes here considered represent two different boundary conditions controlling the whole injection process of a spray of repetitively injected droplets into the combustion chamber. As an unheated spray injection process is here modelled by the adiabatic feeding regime at the mean droplet centre, the isothermal feeding regime at the 
same droplet centre can be viewed as an idealized modelling of a specifically preheated spray injection process. Some studies have shown that the reduction in kinematic viscosity resulting from fuel preheating improves the combustion and emissions performance of the engine [23, 24]. Now, this isothermal feeding regime is here taken into account by supposing the injected fuel at the constant mean temperature $\overline{T_{S}}, T_{S}$ being the spatially uniform although timevarying temperature of saturated vapour at the stabilized mean droplet surface. Indeed, in this stabilized state, actual change in $T_{S}$ value can be considered insignificant and $T_{S}$ is almost equal to $\overline{T_{S}}$ since the mean droplet is maintained at a fixed size. The estimation of the injected fuel temperature $\overline{T_{S}}$ for the isothermal regime will be based on the liquid wet bulb temperature $T_{W B}\left(\overline{T_{S}}=T_{W B}\right)$, given that, $T_{W B}$ is essentially the steady state surface temperature achieved during evaporation of free droplets. The wet bulb temperature should be replaced by the boiling temperature $T_{B}$ only when its estimate is unavailable [20]. Contrary to the classical adiabatic condition which states that the heat flux is null at the spherical droplet centre, the isothermal condition doesn't assure the regularity of the heat flux at the droplet centre. Therefore, the spherical shape of the mean droplet is no more guaranteed in this feeding regime. Nevertheless, we assume for both feeding regimes that the mean droplet remains spherical during the feeding process. Thus, using simplifying assumptions, the present study aims to compare the effect of the two extreme cases bounding the possible range of real liquid fuel inlet temperatures on a subcritical combustion instability phenomenon.

The stabilized mean droplet diameter is supposed sufficiently smaller (about $100 \mu \mathrm{m}$ ) than the wave length, so that the acoustic pressures can be assumed to be temporally undulating but spatially invariant [25]. The present mean droplet configuration is different from that of 
the global equivalence ratio in the spray region by the Sauter Mean Diameter (SMD) used for example in [26] and can be rather linked to the Arithmetic Mean Diameter configuration. This choice is motivated by the analytical approach of the problem since it leads for the mean droplet to an energy conservation equation with fixed boundary conditions. In effect, we consider a stabilized mean spherical droplet, with a constant average radius $\bar{r}_{S}$ within a hot gaseous environment of infinite extent. In these conditions, the actual instantaneous evaporation rate $\dot{M}$ can be assumed nearly equal to its constant mean rate value $\overline{\dot{M}}$ at which the feeding process is realized at the centre. During this process, the density $\rho_{L}$, the specific heat $c_{L}$ and the thermal conductivity $k_{L}$ of the droplet will be treated as constants as assumed in most subcritical evaporation models [21, 27]. For the stabilized state, these fuel properties can be evaluated at some average temperature $T_{\text {Lref }}=0.5\left(T_{0}+T_{W B}\right)$ or $T_{\text {Lref }}=0.5\left(T_{0}+T_{B}\right)$ as in [21] with $T_{0}$ denoting the injected liquid initial temperature at the chamber ambient pressure. The feeding is realized by the same fluid, using a point source located at the centre of the droplet, in such a way that thermal dilatation of the liquid phase is negligible. We will assume that the local feeding rate $\overline{\dot{M}}$ is distributed throughout the droplet as shown in Fig. 1a. Out of the radial thermal convection effect inside the continuously fed droplet from its centre to its evaporation surface, any other convective transport or liquid recirculation phenomenon within the droplet will not be taken into account in the analysis. Only vaporization dynamics will be considered for the gas-phase near the droplet. The influence of combustion will be limited to imposing a stationary composition and temperature at infinity. The combined effects of vaporization dynamics and combustion kinetics, and their eventual retro-action on ambient pressure will not be taken into account here. The mean spherical droplet is assumed to be vaporizing in combustion gases, composed of stoichiometric reaction products. As already mentioned in the introduction, the gas-phase near the droplet 
surface is supposed to evolve in the quasi steady regime and equilibrium conditions at the droplet/gas interface are assumed for the stabilized state. Far from the mean evaporating droplet, the gaseous environment is at constant temperature $T_{C}$ and pressure $P_{C}$. Neither critical nor supercritical phenomena are considered since the system pressure $P_{C}$ is much less than the critical pressure of the liquid. Radiation and second-order effects such as Soret and Dufour effects are also neglected. The boundary conditions for the supplied droplet are shown in Fig. 1b. Subscripts $L$ and $l$ refer to liquid-phase whereas subscript $S$ refers to the condition at the droplet surface.
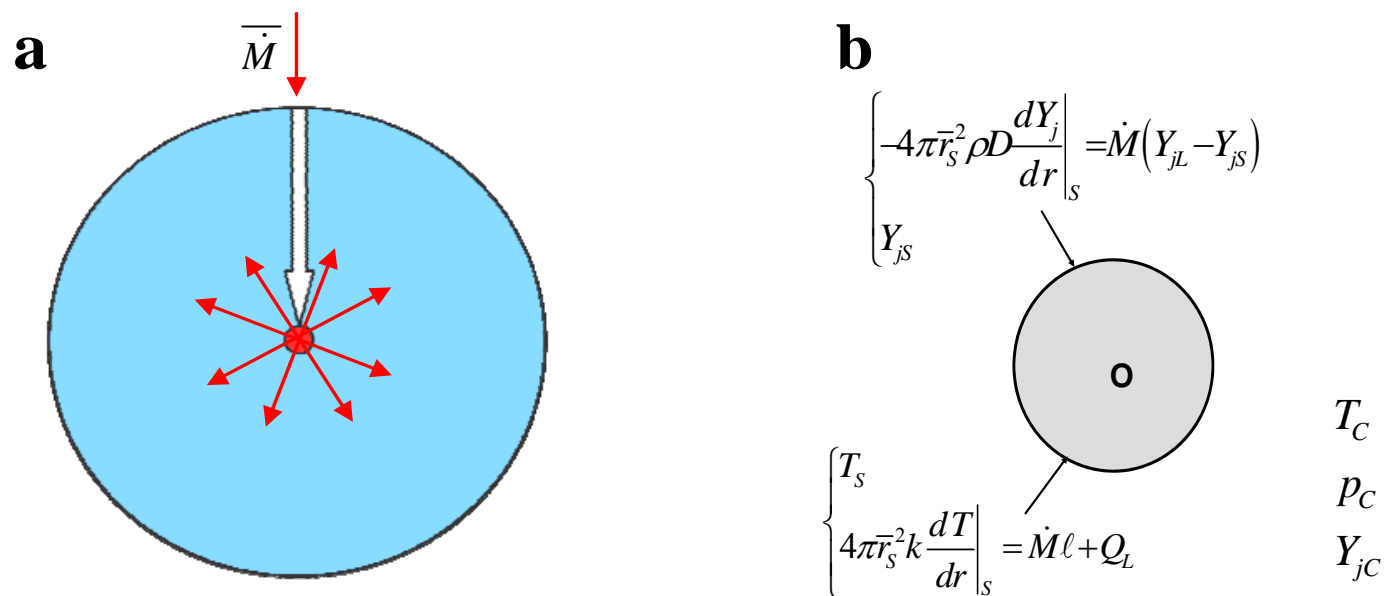

Fig. 1 a The mean vaporizing droplet, continuously fed by a point source placed at its centre. b Boundary conditions for the supplied droplet: parameters $\rho, D$ and $k$ denote respectively the density, the mass diffusion coefficient and the thermal conductivity of the gas-phase. The subscripts $S$ and $C$ refer respectively to the conditions at the droplet surface and at infinity (i.e. the combustion chamber) and $Y_{j}$ designates the mass fraction of jth species.

\subsection{Characteristic times}

The total mass balance of the supplied droplet is:

$$
\frac{d M}{d t}=\overline{\dot{M}}-\dot{M}
$$

where $\overline{\dot{M}}$ is the stationary flow of injection and $\dot{M}$ the instantaneous flow of evaporation of the droplet. In a stabilized regime, one has: $\dot{M} \equiv \overline{\dot{M}}, d M / d t=0$ and $M=\bar{M}$. The residence time of the mean spherical droplet can then be defined as the mean lifetime of an individual 
vaporizing droplet in the array of repetitively injected droplets. Thus, the residence time of the mean droplet corresponds to the residence time of the injected liquid for the droplet as this time replaces the notion of the free droplet lifetime in the present situation of constant volume. The parameter $M$ being the total mass of the supplied droplet and $\bar{M}$ its mean value, the mean residence time is defined as the ratio $\bar{\tau}_{v}=\bar{M} / \overline{\dot{M}}$ of the mass $\bar{M}$ and the stationary feeding rate $\overline{\dot{M}}$. The transfer time by thermal diffusion process is $\bar{\tau}_{T}=\bar{r}_{S}^{2} / \kappa_{L}$, where $\kappa_{L}=k_{L} / \rho_{L} C_{L}$ designates the thermal diffusivity of the liquid. We can thus define the thermal exchange coefficient of the vaporization process as the timescale ratio $\theta=9 \bar{\tau}_{v} / \bar{\tau}_{T}=\bar{\tau}_{v} / \tilde{\tau}_{T}$ (the coefficient 9 permits to obtain later a simple expression of the transfer function). We will also consider the vaporization dynamic of the mean droplet in a pressure fluctuating flow field. Such a problem is very relevant to the analysis of combustion instability in liquid propellant rockets or liquid-fuelled ramjets where the pressure, velocity and gas flow temperature may oscillate in the frequency range of 100-15000 Hz [6]. In order to provide in our present study a parameter that may be used to characterize the instability domain for any fuel, we will consider the reduced frequency defined as $u=3 \omega \bar{\tau}_{v}$ where $\omega$ is the pulsation of ambient pressure harmonic perturbations.

\subsection{Stabilized state equations}

Since the radial thermal convection effect is taken into account, the temperature $T_{l}$ of the liquid is a function of radial coordinate $r$ and time $t$ i.e. $T_{l} \equiv T_{l}(r, t)$ and verifies:

$$
\rho_{L} c_{L} \frac{\partial T_{l}}{\partial t}+\rho_{L} c_{L} v_{r} \frac{\partial T_{l}}{\partial r}-\frac{k_{L}}{r} \frac{\partial^{2}\left(r T_{l}\right)}{\partial r^{2}}=0
$$

where $0<r<\bar{r}_{S}$ with $v_{r}$ the central injection velocity expressed as $v_{r}=\overline{\dot{M}} / 4 \pi \rho_{L} r^{2}$. 
Equation (2) is subject to boundary conditions $\left.\frac{\partial T_{l}}{\partial r}\right|_{0, t}=0$ and $T_{l}\left(\bar{r}_{S}, t\right)=T_{S}(t)$ for the adiabatic injection at the droplet centre or to $T_{l}(0, t)=\bar{T}_{S}$ and $T_{l}\left(\bar{r}_{S}, t\right)=T_{S}(t)$ for the isothermal injection at the droplet centre. The heat $Q_{L}$ transferred into the droplet is given by:

$$
\left.4 \pi \bar{r}_{S}^{2} k_{L} \frac{\partial T_{l}}{\partial r}\right|_{\bar{r}_{s}, t}=Q_{L}=Q-\dot{M} \ell
$$

where $Q$ and $\ell$ are respectively the external gas heat flux and the latent heat of vaporization per unit mass of the liquid. This condition couples the gas and the liquid-phase solutions at the spherical droplet surface.

The gas-phase includes a stabilized mean droplet surface in local evaporation equilibrium with an ideal gas mixture evolving in a quasi-steady regime. The instantaneous mass vaporization rate from the droplet surface can be calculated using the following equations:

$$
\dot{M}=2 \pi \rho D r_{S} S h^{*} \ln \left(1+B_{M}\right)=4 \pi \frac{k}{c_{p}} r_{S} N u^{*} \ln \left(1+B_{T}\right)
$$

with the Spalding parameter for mass exchange $B_{M}=\left(Y_{F S}-Y_{F C}\right) /\left(1-Y_{F S}\right)$ and for heat exchange $B_{T}=c_{p}\left(T_{C}-T_{S}\right) /\left(\ell+Q_{L} / \dot{M}\right)$. The gas-phase properties are: the density $\rho$, the mass diffusion coefficient $D$, the thermal conductivity $k$ and the specific heat at constant pressure $c_{p}$. The Sherwood $S h^{*}$ and Nusselt $N u^{*}$ numbers were introduced by Abramzon and Sirignano in their extended film model [21]. The mass fraction of species $j$ is denoted $Y_{j}$ and $T_{C}$ is the ambient temperature. The subscripts $F, S$, and $C$, represent respectively the fuel, the droplet surface and the conditions far from the droplet. The saturated vapour pressure $p_{\text {sat }}\left(T_{S}\right)=\exp \left(a-b /\left(T_{S}-c\right)\right)$, (with related coefficients $a, b$ and $c$ ), is connected to the mole fraction of the droplet vapour at the droplet surface by the relation $p X_{F S}=p_{\text {sat }}\left(T_{S}\right)$, where $p$ is the ambient pressure, $X_{j}$ the mole fraction of species $j$ for the ideal gas mixture. 
Then, we can relate the mass fraction $Y_{F S}$ of the droplet vapour at the droplet surface to the mole fraction $X_{F S}$ :

$$
Y_{F S}=\frac{\mathrm{M}_{F}}{\mathrm{M}_{F} X_{F S}+\mathrm{M}_{A} X_{A S}} X_{F S}
$$

where $\mathrm{M}_{j}$ is the molecular weight of species $j$ (the gaseous mixture is made up of fuel species $F$ and diluted species $A$ while the liquid is of only fuel species $F$ ). Given that temperature and concentrations are not constant in the environment of the droplet, the averaged properties can be evaluated at some reference temperature $\bar{T}=T_{S}+A_{r}\left(T_{C}-T_{S}\right)$ and composition $\bar{Y}_{F}=Y_{F S}+A_{r}\left(Y_{F C}-Y_{F S}\right)$, where $A_{r}=1 / 3$ will be chosen. The calculations for linear analyses were performed [15] with both Sherwood and Nusselt numbers taken equal to 2 and Lewis number taken equal to unity.

\section{Linear Analyses for Small Perturbations}

Our objective is to build an approximate analytical model of reference for the harmonic perturbations in pressure. To this end, we will supplement the previous developments of the models of the continuously fed spherical droplet [13-16] by taking into account the not yet studied radial thermal convection effect on the process, for both adiabatic and isothermal feeding cases.

\subsection{Linear analysis of the liquid-phase equations}

The velocity perturbation is assumed equal to zero. The wave pulsation $\omega$ accounts for the acoustic influence on the evaporation rate and the internal temperature perturbations since all the derived solutions are functions of the reduced frequency $u=3 \omega \bar{\tau}_{v}$. Introducing then small harmonic perturbations of pulsation $\omega$ of the form $f^{\prime}=(f-\bar{f}) / \bar{f}=\hat{f}(r) \exp (i \omega t)$, the ambient constant pressure $p=\overline{p_{C}}$ will take the perturbed form $p^{\prime}=\hat{p}_{C} \exp (i \omega t)$. We set 
$T_{l}^{\prime}(r, t)=\left(\bar{T}_{l}(r, t)-T_{l}(r, t)\right) / \bar{T}_{l}(r, t)=\hat{T}_{l}(r) \exp (i \omega t)$ and $\Delta Q_{L}=\Delta \hat{Q}_{L}(r) \exp (i \omega t)$. Equation

(2) can then be transformed to:

$$
i \omega \bar{\tau}_{T} \xi \hat{T}_{l}+\frac{1}{3 \theta \xi} \frac{d \hat{T}_{l}}{d \xi}-\frac{d^{2}\left(\xi \hat{T}_{l}\right)}{d \xi^{2}}=0
$$

where, instead of the radial variable $r\left(0<r<\bar{r}_{S}\right), \hat{T}_{l}$ is rather taken as a function of the reduced radius variable $\xi=r / \bar{r}_{S}(0<\xi<1)$. In connection with $\xi$, the boundary conditions in the adiabatic feeding regime are :

$$
\left\{\begin{array}{l}
\left.\frac{d \hat{T}_{l}}{d \xi}\right|_{\xi=0}=0 \\
\hat{T}_{l}(1)=\hat{T}_{S}
\end{array}\right.
$$

and become in the isothermal feeding regime:

$$
\left\{\begin{array}{l}
\hat{T}_{l}(0)=0 \\
\hat{T}_{l}(1)=\hat{T}_{S}
\end{array}\right.
$$

We now consider the two conjugate complex numbers $s_{0}=(1+i)\left(\omega / 2 \kappa_{L}\right)^{\frac{1}{2}}$ and $\bar{s}_{0}=(1-i)\left(\omega / 2 \kappa_{L}\right)^{\frac{1}{2}}, s_{0}$ and $-s_{0}$ being the two complex roots of the characteristic equation $i \omega-\kappa_{L} s^{2}=0$ obtained from Eq. (6) when neglecting the convective term $\frac{1}{3 \theta \xi} \frac{d \hat{T}_{l}}{d \xi}$. In the adiabatic centre case a solution of Eq. (6) can be sought in the form of $\xi \hat{T}_{l}(\xi)=G(\xi)\left(1-\cos \left(\bar{s}_{0} \bar{r}_{S} \xi\right)\right)$ while in the isothermal feeding case a solution will be sought in the form of $\xi \hat{T}_{l}(\xi)=H(\xi)\left(1-\cos \left(s_{0} \bar{r}_{S} \xi\right)\right)$, with $G$ and $H$ referring to functions to be determined. Therefore, using in the neighbourhood of $\xi=0$, the second-order truncated expansions of sine and cosine functions that are $\sin \left(\bar{s}_{0} \bar{r}_{S} \xi\right) \simeq \bar{s}_{0} \bar{r}_{S} \xi$ and $\cos \left(\bar{s}_{0} \bar{r}_{S} \xi\right) \simeq 1-\left(\bar{s}_{0} \bar{r}_{S} \xi\right)^{2} / 2$, we deduce that the functions $\xi G$ and $\xi H$ must respectively verify the following second order equations:

$$
\xi^{2} \frac{d^{2}(\xi G)}{d \xi^{2}}+\left(2 \xi-\frac{3}{\theta}\right) \frac{d(\xi G)}{d \xi}=0
$$

and 


$$
\xi^{2} \frac{d^{2}(\xi H)}{d \xi^{2}}+\left(2 \xi-\frac{3}{\theta}\right) \frac{d(\xi H)}{d \xi}-2 s_{0}^{2} \bar{r}_{s}^{2} \xi^{2}(\xi H)=0
$$

Equations (10) and (9) are respectively a double confluent Heun equation and its degenerate case. By using the boundary conditions Eq. (7) in the adiabatic centre regime, an approximate analytical solution of Eq. (6) can be expressed:

$$
\hat{T}_{l}(\xi)=\frac{\hat{T}_{S} \exp \left(\frac{3}{2 \theta}\left(1-\frac{1}{\xi}\right)\right)\left(1-\cos \left(\bar{S}_{0} \bar{r}_{S} \xi\right)\right)}{\left(1-\cos \left(\bar{s}_{0} \bar{r}_{S}\right)\right) \xi^{2}}
$$

Adopting now the notation of the Maple manual, a particular solution of Eq. (10) over the open range $0<\xi<1$ (isothermal feeding case) can be expressed as: $\mathrm{H}(\xi)=C_{2} \exp (-3 / \theta \xi) \operatorname{HeunD}\left(x_{1}, x_{2}, x_{3}, x_{4},\left(\xi^{2}-1\right) /\left(\xi^{2}+1\right)\right) / \xi^{\frac{3}{2}}$ where $C_{2}$ is an arbitrary constant and $\operatorname{HeunD}\left(x_{1}, x_{2}, x_{3}, x_{4},\left(\xi^{2}-1\right) /\left(\xi^{2}+1\right)\right)$ is the double confluent Heun function with the corresponding four parameters: $x_{1}=0, x_{2}=-\left(\theta^{2}-9-24 i u \theta\right) / 4 \theta^{2}$, $x_{3}=3(8 i u \theta-3) / 2 \theta^{2}$ and $x_{4}=\left(\theta^{2}+9+24 i u \theta\right) / 4 \theta^{2}$. We recall that the parameter $u=3 \omega \bar{\tau}_{v}$ is the reduced frequency previously defined in subsection 2.2. Finally, by using the boundary conditions in the isothermal feeding regime i.e. Eq. (8), an approximate analytical solution of Eq. (6) can be expressed in the form:

$$
\hat{T}_{l}(\xi)=\left\{\begin{array}{l}
0, \xi=0 \\
\frac{\hat{T}_{S} \exp \left(\frac{3}{2 \theta}\left(1-\frac{1}{\xi}\right)\right)\left(1-\cos \left(s_{0} \bar{r}_{S} \xi\right)\right) \operatorname{HeunD}\left(x_{1}, x_{2}, x_{3}, x_{4}, \frac{\xi^{2}-1}{\xi^{2}+1}\right)}{\left(1-\cos \left(s_{0} \bar{r}_{S}\right)\right) \xi^{\frac{5}{2}}}, 0<\xi \leq 1
\end{array}\right.
$$

Since it oscillates indefinitely as it approaches $\xi=0$ by the positive direction, the approximate analytical solution of the isothermal centre case presents an essential discontinuity at $\xi=0$ contrary to that of the adiabatic centre case Eq. (11) which is rather regular at $\xi=0$. In fact, neither the continuity nor the regularity of the function $\hat{T}_{l}$ at this centre point do intervene in the calculation of the mass response factor which is of our 
concern, but only the regularity conditions at the droplet surface $\xi=1$ are needed. These regularity conditions are well verified by both approximate solutions Eq. (11) and Eq. (12). Thus, for both feeding regimes, the flow condition at the droplet surface Eq. (3) which now reads $\left.4 \pi \bar{r}_{S} k_{L} \bar{T}_{S} \frac{d \hat{T}_{l}}{d \xi}\right|_{\xi=1}=\Delta \hat{Q}_{L}$ can apply to the derived approximate solutions. That leads to:

$$
\Delta \hat{Q}_{L}=-4 \pi \bar{r}_{S} k_{L} \bar{T}_{S} E(u, \theta) \hat{T}_{S}
$$

where $E$ is a function of $u$ and $\theta$. Hence, we found for the adiabatic feeding case:

$$
E(u, \theta)=\frac{\bar{s}_{0} \bar{r}_{S} \theta \sin \left(\bar{s}_{0} \bar{r}_{S}\right)+2 \theta \cos \left(\bar{s}_{0} \bar{r}_{S}\right)-3 \cos \left(\bar{s}_{0} \bar{r}_{S}\right)-2 \theta+3}{\theta\left(1-\cos \left(\bar{s}_{0} \bar{r}_{S}\right)\right)}
$$

whereas for the isothermal feeding case, calculations yield:

$$
E(u, \theta)=-\frac{1}{2} \frac{2 \theta s_{0} \bar{r}_{S} \sin \left(s_{0} \bar{r}_{S}\right)+5 \theta \cos \left(s_{0} \bar{r}_{S}\right)-3 \cos \left(s_{0} \bar{r}_{S}\right)-5 \theta+3}{\theta\left(1-\cos \left(s_{0} \bar{r}_{S}\right)\right)}
$$

with $s_{0} \bar{r}_{S}=(1+i)(3 u / 2 \theta)^{\frac{1}{2}}, \bar{s}_{0} \bar{r}_{S}=(1-i)(3 u / 2 \theta)^{\frac{1}{2}}, u=3 \omega \bar{\tau}_{v}$ and $\theta=\bar{\tau}_{v} / \tilde{\tau}_{T}$.

\subsection{Linear analysis of the gas-phase equations}

We will now recall the linearized equations for the liquid/gas interface used in $[15,16]$. At the stabilized state, any thermodynamic variable $f$ of the gas-phase has a uniform distribution $\bar{f}$. Introducing small harmonic perturbations $f^{\prime}=\hat{f}(r) \exp (i \omega t)$, we look for the relation between the imposed chamber perturbation $p^{\prime}=\hat{p}_{C} \exp (i \omega t)$ and the resulting mass flow rate perturbation $\dot{M}^{\prime}=\hat{\dot{M}} \exp (i \omega t)$. From the equations of the gas-phase (see subsection 2.3), it was deduced that [15]:

$$
\hat{\dot{M}}=\alpha \frac{i u}{1+i u}\left(\bar{b} \hat{T}_{S}-\hat{p}_{C}\right)
$$

with $u=3 \omega \bar{\tau}_{v}, \dot{M}^{\prime}=\Delta \dot{M} / \overline{\dot{M}}=\hat{\dot{M}} \exp (i \omega t)$ and:

$$
\Delta \hat{Q}_{L}=\overline{\dot{M}} \bar{\ell}\left(\bar{a} \hat{p}_{C}-\mu \hat{T}_{S}\right)
$$


where $\Delta Q_{L}=Q_{L}-\bar{Q}_{L}=Q_{L}=\Delta \hat{Q}_{L} \exp (i \omega t)$ as $\bar{Q}_{L}=0$. The coefficients involved in Eq. (16) and Eq. (17) are expressed as follows:

$$
\left\{\begin{array}{l}
\bar{a}=\frac{\bar{T}_{C}}{\bar{T}_{C}-\bar{T}_{S}} \frac{\gamma-1}{\gamma}+\varphi, \quad \bar{b}=\frac{\bar{T}_{S}}{\left(\bar{T}_{S}-c\right)^{2}} b, \\
\mu=\frac{\bar{T}_{S}}{\bar{T}_{C}-\bar{T}_{S}}-\frac{2 c}{\bar{T}_{S}-c}+\bar{b} \varphi, \\
\alpha=\frac{\bar{B}_{M}}{\left(1+\bar{B}_{M}\right) \ln \left(1+\bar{B}_{M}\right)} \frac{\bar{Y}_{A C} \bar{Y}_{F S}}{\bar{Y}_{A S}\left(\bar{Y}_{F S}-\bar{Y}_{F C}\right)} \frac{\mathrm{M}_{F}}{\mathrm{M}_{F} \bar{X}_{F S}+\mathrm{M}_{A} \bar{X}_{A S}}
\end{array}\right.
$$

In these expressions, $\gamma$ stands for the isentropic coefficient (assumed to be constant), the function $\varphi$ equals the quantity $\varphi=\bar{Y}_{A C} \bar{Y}_{F S} \mathrm{M}_{F} / \bar{Y}_{A S}\left(\bar{Y}_{F S}-\bar{Y}_{F C}\right)\left(\mathrm{M}_{F} \bar{X}_{F S}+\mathrm{M}_{A} \bar{X}_{A S}\right)$ and the latent heat is given in the form: $\ell=b R T_{S}^{2} / \mathrm{M}_{F}\left(T_{S}-c\right)^{2}$ with $R$ denoting the universal gas constant.

\subsection{Mass response factor}

The response factor $N$ is expressed as the ratio of the magnitude of heat or mass perturbation to the magnitude of the pressure perturbation and thus, includes phase relations. The reduced pressure perturbation is defined as $p^{\prime}=(p-\bar{p}) / \bar{p}$, and the resulting reduced heat or mass perturbation is $q^{\prime}=(q-\bar{q}) / \bar{q}$. The response factor $N$ is thus defined as:

$$
N=\frac{\iint_{V, t} q^{\prime}(V, t) p^{\prime}(V, t) d t d V}{\iint_{V, t}\left(p^{\prime}(V, t)\right)^{2} d t d V}
$$

where the double integral value is calculated over a given period of time $t$ in a finite volume $V$. For sinusoidal oscillations which are uniform over a finite volume, the response factor can be reduced to $N=(|\hat{q}| /|\hat{p}|) \cos \phi$, where $|\hat{q}|$ and $|\hat{p}|$ are the moduli and $\phi$ the phase difference between $q^{\prime}$ and $p^{\prime}$. We now consider the complex transfer function $Z=\hat{\dot{M}} /\left(\alpha \hat{p}_{C}\right)$ whose expression here is brought about using Eq. (13), Eq. (16) and Eq. (17):

$$
Z(\theta, u)=\frac{i u}{1+i u} \frac{A+\theta E(u, \theta)}{B-\theta E(u, \theta)}
$$


The parameters $A=3(\bar{a} \bar{b}-\mu) / \lambda$ and $B=3 \mu / \lambda$ are thermodynamic coefficients related to the fuel physical properties with $\lambda=c_{L} \bar{T}_{S} / \bar{\ell}$. The mass response factor $N$ can equally be deduced as the real part of the transfer function $Z$. From now down this paper, we will consider and call "response factor", the reduced response factor, which is the real part of the transfer function $Z$ :

$$
\frac{N}{\alpha}=\Re(Z)
$$

The response factor is assumed positive when the vaporization rate and the chamber pressure are either above or below their mean values. But when the vaporization rate and the chamber pressure are on the opposite sides of their means it is then assumed negative [18]. Hence, the phase difference $\phi$ between the vaporization rate and the chamber pressure, defined as $\phi=\arg (Z)$, appears to be a key parameter in pressure-related-oscillations analyses. This phase angle was shown to remain insensitive to the chamber mean pressure magnitude [3]. We now briefly recall, concerning the pure conduction model of the spherical mean droplet $[15,16]$, that the complex transfer function $Z$ has the same expression as laid in Eq. (19). In that model, the expression of the function $E$ for the adiabatic injection case was $E(u, \theta)=1-s_{0} \bar{r}_{S} \operatorname{coth}\left(s_{0} \bar{r}_{S}\right)$ whereas for the isothermal injection case calculations yielded $E(u, \theta)=1+\bar{s}_{0} \bar{r}_{S} \tan \left(\bar{s}_{0} \bar{r}_{S}\right)$ with the same parameters as in the present model: $s_{0} \bar{r}_{S}=(1+i)(3 u / 2 \theta)^{\frac{1}{2}}, \bar{s}_{0} \bar{r}_{S}=(1-i)(3 u / 2 \theta)^{\frac{1}{2}}, u=3 \omega \bar{\tau}_{v}$ and $\theta=\bar{\tau}_{v} / \tilde{\tau}_{T}$.

\section{Results and discussion}

As for the pure conduction models $[15,16]$, calculations and curves are performed in the present analysis with the thermodynamic coefficients $A=10$ and $B=100$. These values of $A$ and $B$ correspond approximately to orders of magnitude of values encountered in the classical fuels [28]. With respect to the two feeding regimes, the treatment of the data will 
consist to relate the mass response factor to the effects respectively of the radial thermal convection and of the process characteristic times and again of the thermodynamic coefficients $A$ and $B$. Figure 2 shows, for both adiabatic and isothermal feeding regimes, response factor curves as functions of the reduced frequency $u=3 \omega \bar{\tau}_{v}$ for arbitrary values of the exchange coefficient $\theta=\bar{\tau}_{v} / \tilde{\tau}_{T}$. For comparison purposes, corresponding response factor curves in the pure conduction model are reproduced in Fig. 3.

\subsection{General remarks}

In the adiabatic centre regime (Fig. 2), the response factor curve shows always a positive response region corresponding to instability domain. As in the pure conduction model (Fig. 3), a typical response factor curve arises from zero at the lowest frequency, exhibits a peak value around a fixed reduced frequency $u_{p} \cong 3$ and then decreases and later on takes negative values at higher frequencies. The cut-off reduced frequency $u_{c}$, corresponding to a zero response factor, is to be considered as a critical frequency since it divides the frequency response into regions of destabilizing and stabilizing influences. When $\theta$ increases from 1 on, the critical frequency $u_{c}$ tends to decrease quickly first, then reaches a minimum value for $\theta$ about 30 and then again begins to increase very slowly to finally attain a limit frequency value slightly greater than 55 (Figs. 2c and 2e). In fact, in this feeding regime, and even for the pure conduction model, once $\theta>150$, all the response factor curves tend to collapse into a single line (Figs. 2e and 3e).

Concerning the isothermal centre regime, as in the pure conduction model, existence of cutoff frequency $u_{c}$ depends closely on values of $\theta$. When $\theta$ increases from 1 to a value about 200 , the critical frequency $u_{c}$ varies quite quickly between 20 and a value about 65 , then completely vanishes on the layout for higher values of $\theta(\theta \geq 225)$. Compared with the pure 

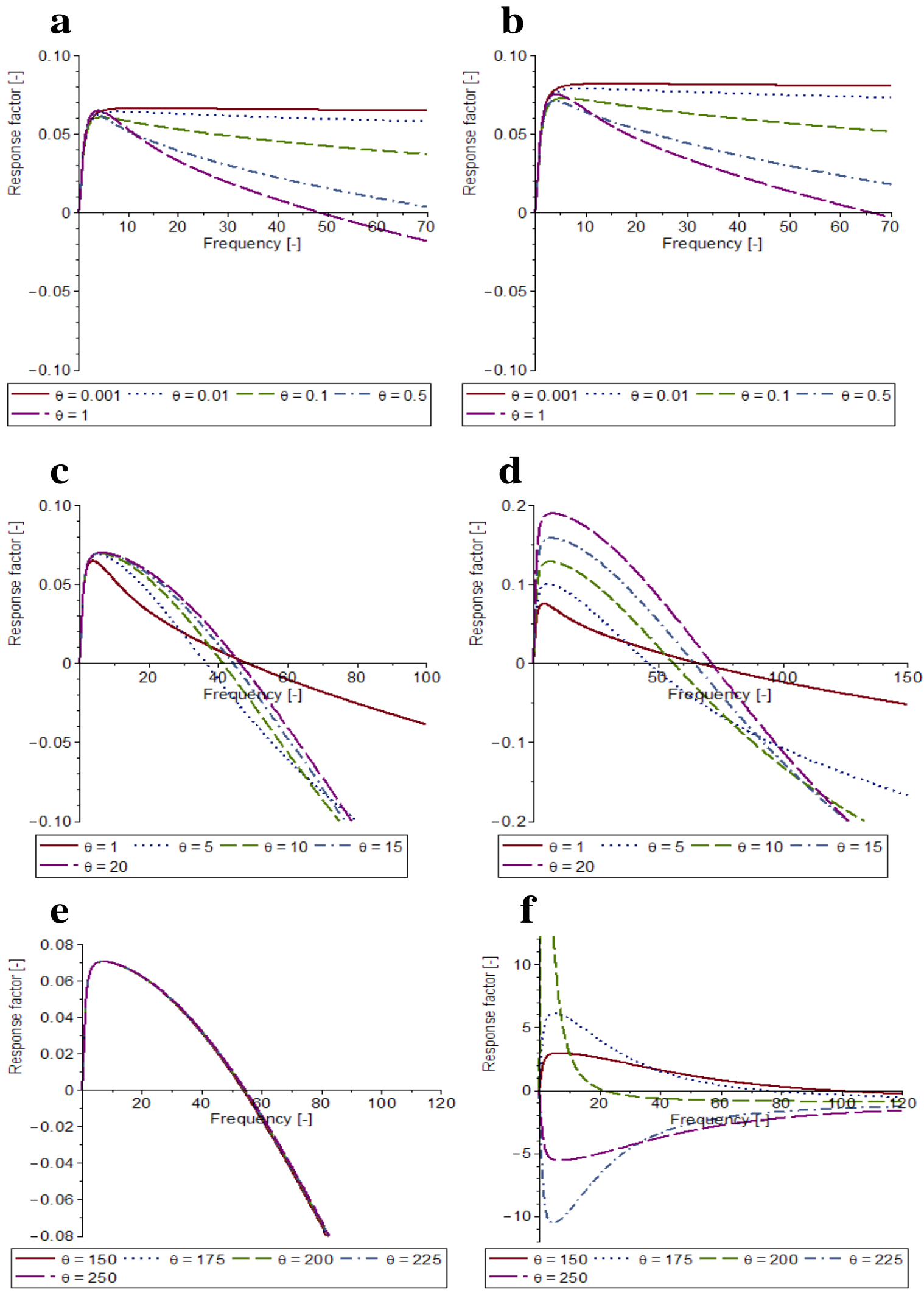

Fig. 2 Influence of reduced exchange coefficient $\theta$ on the response factor $N / \alpha=\Re(Z)$ for the mean spherical droplet model with thermal convection effect; $A=10$ and $B=100$. a Adiabatic centre. b Isothermal centre. c Adiabatic centre. d Isothermal centre. e Adiabatic centre. f Isothermal centre 
a

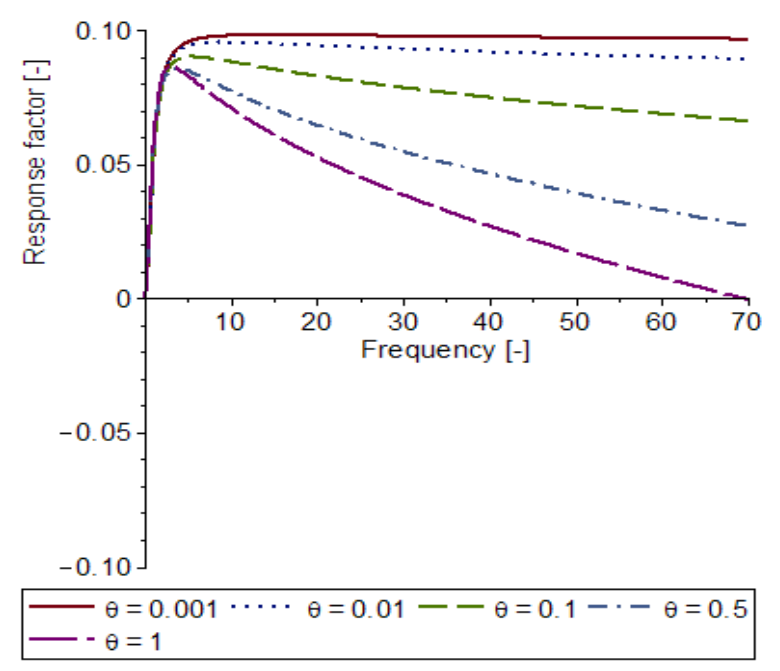

C

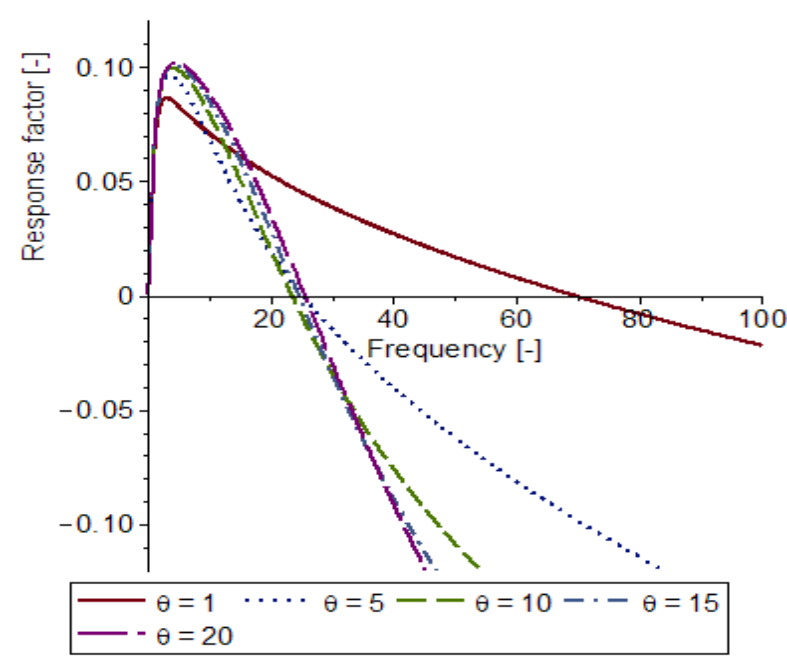

$\mathbf{e}$

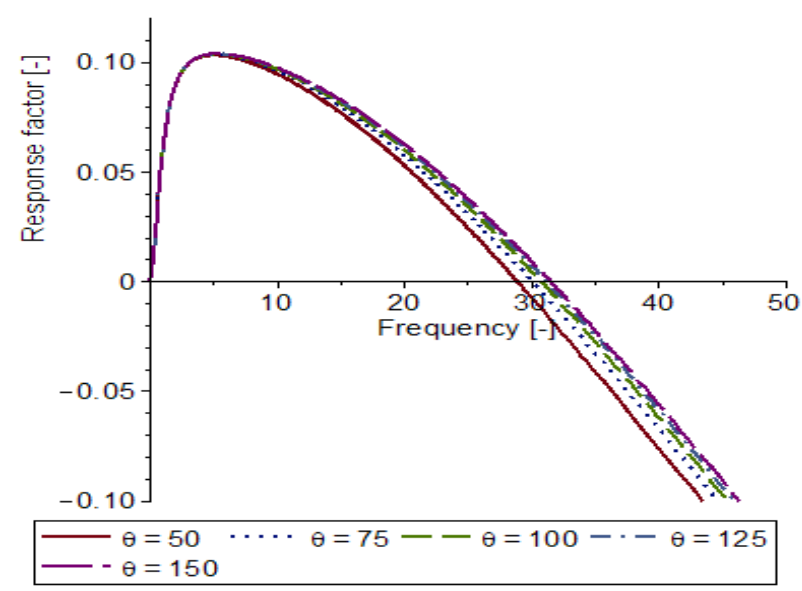

b

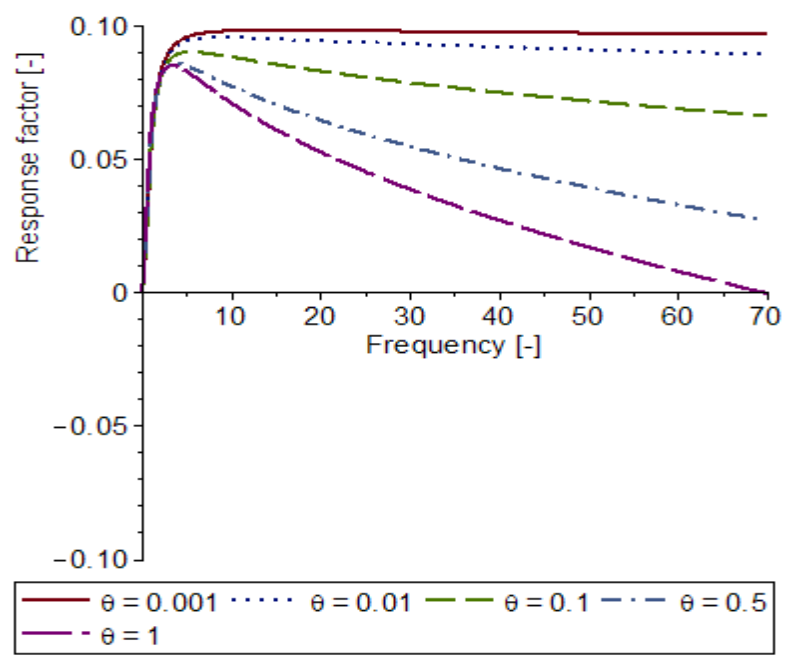

d
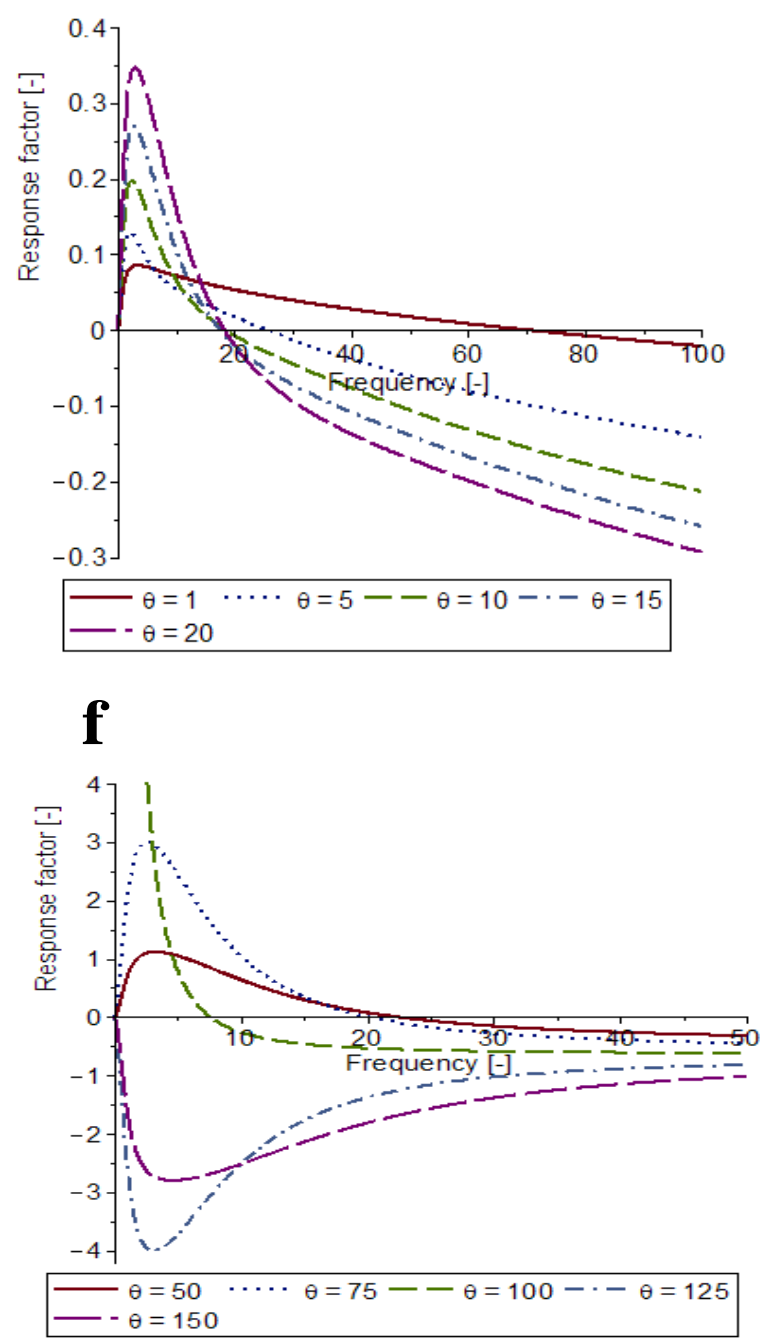

Fig. 3 Influence of reduced exchange coefficient $\theta$ on the response factor $N / \alpha=\Re(Z)$ for the mean spherical droplet model without thermal convection effect (pure conduction model); $A=10$ and $B=100$. a Adiabatic centre. $\mathbf{b}$ Isothermal centre. c Adiabatic centre. $\mathbf{d}$ Isothermal centre. e Adiabatic centre. f Isothermal centre 
conduction model, the critical frequency values are greater whereas the positive peak value stand always at about the same frequency $u_{p} \cong 3$. Also, for the model with consideration to thermal convection effect, the peak value grows very quickly and seems to tend to infinity for a value of $\theta$ about 200. In fact, larger peak values growing beyond the unity value appear for $100<\theta \leq 200$. Especially, for $\theta$ about 200 the frequency response curve for isothermal feeding regime asymptotically diverges from unity to infinity, along the vertical line passing at $u_{p}$. But curves for higher values of $\theta(\theta \geq 225)$ show negative response factors for all frequencies. This behaviour is globally similar to that observed in the pure conduction model (Figs. $2 \mathrm{f}$ and $3 \mathrm{f}$ ) except that more large values of critical frequencies $u_{c}$ and greater relative exchange coefficient $\theta$ appear in the present model.

In both adiabatic and isothermal feeding regimes, the frequency response curves are quite similar for relatively small values of the exchange coefficient $(0<\theta \leq 1)$ as shown in Figs. 2a and $2 \mathrm{~b}$, except that the isothermal feeding regime presents more large cut-off frequency values and consequently more large instability domains. For both regimes, the peak responses seem to be at a same specific frequency $u_{p}$, which is relatively unaffected by the variation of $\theta$. The main differences in the frequency response for the isothermal injection regime compared with the adiabatic one are the larger peak values of the response factor at the particular frequency $u_{p}$ and the absence of a positive response region when $\theta \geq 225$.

\subsection{Effects of the radial thermal convection}

First, in the adiabatic feeding regime, when $0<\theta \leq 1$, response factor curves of the mean spherical droplet model with thermal convection effect show, against all expectations, lower cut-off frequencies than that of the pure conduction model (Figs. 2a and 3a). This fact can be readily related to the nature of the adiabatic feeding regime since, in this regime, the thermal wave propagation from the droplet surface to its centre is counterbalanced by the radial 
thermal convection effect from the droplet centre to the surface. When the exchange coefficient $\theta$ is low, that is $\bar{\tau}_{v}<<\bar{\tau}_{T}$, the thermal convection effect can be considered more important than that of the thermal conduction, and being in the adiabatic feeding regime, the temperature gradient $\frac{\partial \hat{T}_{l}}{\partial \xi}$ is null at the droplet centre. To the contrary, given that $\frac{\partial \hat{T}_{l}}{\partial \xi}$ is well above 0 at the droplet centre in the isothermal feeding regime, the thermal convection will rather help the mean spherical droplet to quickly hold its thermal equilibrium, favouring therefore the thermal wave penetration inside the droplet and the vaporization rate response.

Considering again the adiabatic feeding regime, a response factor curve shows necessary a cut-off frequency $u_{c}$ depending on the value of the exchange coefficient $\theta$. In this feeding regime, calculations show that

$$
\frac{N}{\alpha} \rightarrow \frac{(4 A B+14 A-10 B-36) u^{2}-u^{4}}{36+24 B+4 B^{2}+\left(37+24 B+4 B^{2}\right) u^{2}+u^{4}}
$$

when $\theta \rightarrow \infty$, that is $\bar{\tau}_{T}<<\bar{\tau}_{v}$ (the thermal convection effect is no more dominant).

Therefore the critical frequency tends to a constant value $u_{c} \rightarrow \sqrt{4 A B+14 A-10 B-36}$ $\cong 55.7$ for $A=10$ and $B=100)$. These values can be compared with the limit values obtained in the same feeding regime for the pure conduction models, which are [29]:

$$
\frac{N}{\alpha} \rightarrow \frac{(A+B+A B) u^{2}-u^{4}}{B^{2}+\left(1+B^{2}\right) u^{2}+u^{4}}
$$

and $u_{c} \rightarrow \sqrt{A+A B+B}(\cong 33.3$ for $A=10$ and $B=100)$ when $\theta \rightarrow \infty$. In fact, once $\theta>1$, the radial thermal convection effect strongly affects the process by extending considerably the positive response domain even if this convection effect remains unimportant in the system. 
Considering now the isothermal feeding regime, the response factor curve may show negative response for all frequencies provided that $\theta$ is sufficiently large $(\theta \geq 225)$. This fact is confirmed by theoretical consideration since, in this feeding regime, calculations give:

$$
\frac{N}{\alpha} \rightarrow \frac{-u^{2}}{1+u^{2}}
$$

when $\theta \rightarrow \infty$. Indeed, in this feeding regime, the limit function (23) is identical to that of the pure conduction model of the mean spherical droplet and even to that of the pastille-shaped droplet model with or without respect for the thermal convection effect [29]. In short, in both adiabatic and isothermal regimes, the taking into account of the thermal convection effect once $\theta>1$, strongly affects the mass response factor by enlarging notably, whenever it exists, the scope of the instability domain.

\subsection{Effects of process characteristic times}

It has been shown (1994) that, in the frequency range of interest in ramjet combustion instabilities $(100-8000 \mathrm{~Hz})$, the droplet lifetime and the period of pressure oscillation can be of the same order of magnitude [7]. Indeed, in accordance with previous studies of the mean spherical droplet in the adiabatic feeding regime and even through the analysis of the pastilleshaped droplet subjected to the same regime [13-16, 29], the response factor peak value always occurs at the same reduced frequency of about $u=3$, that is $u_{p}=3 \omega \bar{\tau}_{v} \cong 3$ or $\omega \bar{\tau}_{v} \cong 1$

. Now, in isothermal centre cases (Figs. $2 \mathrm{~b}, 2 \mathrm{~d}, 2 \mathrm{f}$ and $3 \mathrm{~b}, 3 \mathrm{~d}, 3 \mathrm{f}$ ), a positive peak value of the response factor, when it exists, appears around the same frequency $u_{p}$. According to Rayleigh criterion, this reduced frequency $u_{p}=3 \omega \bar{\tau}_{v} \cong 3$, at which the droplet lifetime (injected liquid residence time $\bar{\tau}_{v}$ ) matches the period of pressure oscillations $1 / \omega$, induces a positive peak response factor because it favours mass transfer in phase with pressure oscillation. Thus, regardless of the type of the feeding regime, whenever positive responses appear in the 
system, the vaporization rate can fully respond to the acoustic oscillations only when the droplet lifetime equals the period of ambient pressure oscillations.

Following Rayleigh criterion, the combined effect of the three process characteristic times (thermal diffusion time $\bar{\tau}_{T}=\bar{r}_{S}^{2} / \kappa_{L}$, droplet residence time $\bar{\tau}_{v}$ and period of pressure oscillations $1 / \omega)$ can be envisaged in the light of the well-known time-lag model $[4,6,7]$. Figure 4 shows, for both adiabatic and isothermal feeding regimes, phase-angle curves as functions of the reduced frequency $u=3 \omega \bar{\tau}_{v}$ for arbitrary values of the exchange coefficient $\theta=\bar{\tau}_{v} / \tilde{\tau}_{T}$. It appears that, all the above-mentioned trends concerning Fig. 2 are readily confirmed by the curve profiles obtained in Fig. 4. In the adiabatic feeding regime (Fig. 4a) phase-angle curves collapse in a single line once $\theta \geq 1$, accounting therefore for response factor curves profiles obtained in Figs. 2a, 2c, 2e. More importantly, the phase-angle curves unique cut-off frequency can be precisely approximated to the peak frequency $u_{p}=3 \omega \bar{\tau}_{v} \cong 3$ at which the vaporization rate oscillates perfectly in phase with the acoustic pressure $(\phi=0)$. The curve profiles observed for the isothermal feeding regime (Fig. 4b) are also highly suggestive, in comparison with the profiles obtained in Figs. 2b, 2d, 2f. Indeed, considering $\theta \leq 200$, the phase lag $\phi$ decreases from $\pi / 2$ to a slightly inferior value when the frequency $u$ increases from 0 to about the peak frequency $u_{p}$. And then, $\phi$ increases slowly till the maximum value of $\pi$, expressing therefore a progressive damp of instability in the system. Now, for $\theta=250(\theta>200)$, the phase-lag curve exhibits, about the frequency $u_{p}$, an instantaneous change from the extreme value $-\pi$ to the other extreme value $\pi$, accounting therefore for the intriguing changes noted in the related response factor curves profiles (Fig. 2f). In practice, the residence time depends on the mean droplet diameter size, the thermal diffusion time depends on the propellant diffusivity, and the period of the oscillation can be 
related to the shape of the combustion chamber. The judicious choice of these parameter values in agreement with the above results may permit to obtain more stability in propellant combustion systems.
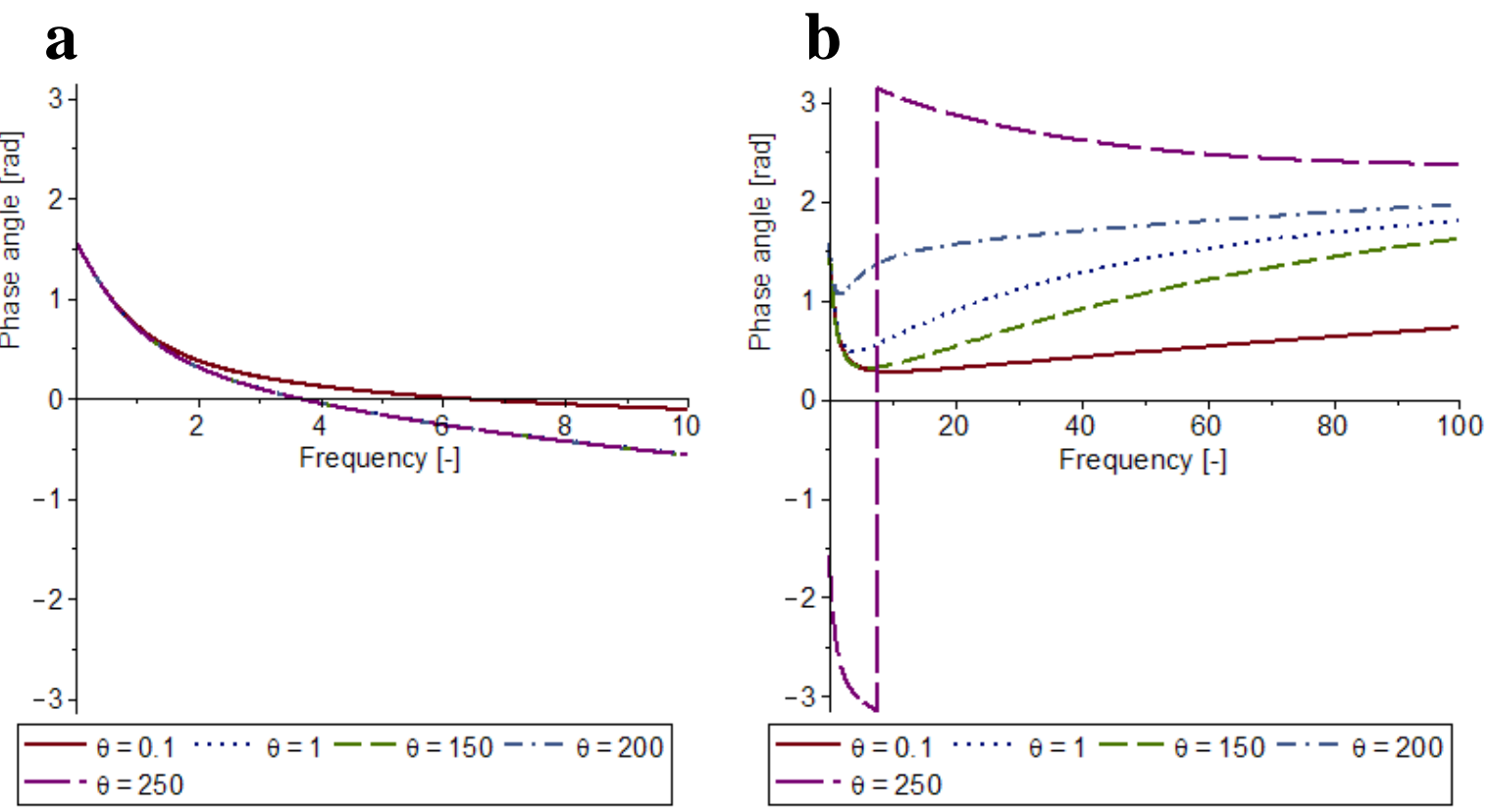

Fig. 4 Influence of reduced exchange coefficient $\theta$ on phase lags $\phi$ of vaporization rate with regard to acoustic pressure for the mean spherical droplet model with thermal convection effect; $A=10$ and $B=100$. a Adiabatic centre. $\mathbf{b}$ Isothermal centre

\subsection{Effects of the thermodynamic coefficients $A$ and $B$}

Unlike the adiabatic feeding case, curves in the isothermal feeding regime (Fig. 2f) show intriguing changes in their profiles, once $\theta$ gets superior to a certain value $\theta_{d}$. An explanation given above indicates a rapid realisation of the thermal equilibrium inside the mean droplet in the isothermal feeding regime, and then suggests possible influences of fuel physical properties effects in the system. From this point of view and in order to determine the specific value $\theta_{d}$, the ratio $x=u / \theta=\omega \bar{\tau}_{T} / 3$ may be particularly useful in this analysis in as much as the residence time $\bar{\tau}_{v}$ does not intervene in it. But, the thermal diffusion time $\bar{\tau}_{T}$ and the pulsation of the oscillating wave $\omega$ intervene. Therefore, taking this ratio negligible at the fixed peak frequency $u_{p}=3 \omega_{p} \bar{\tau}_{v}^{p} \cong 3$ comes to taking the thermal transfer time by diffusion $\bar{\tau}_{T}$ negligible compared to either the oscillation period $\Gamma_{p}=1 / \omega_{p}$ or the residence time $\bar{\tau}_{v}^{p}$. 
Thence, as in [16], an estimation of the complex transfer function $Z$ (Eq. (19)) can be obtained in the neighbourhood of $x=0$ by a limited expansion according to the ratio $x=u / \theta$ while assuming $u$ closer to $u_{p} \cong 3$. As this ratio can be supposed negligible in the expression of the complex transfer function $Z$ provided that $\theta$ is taken sufficiently large, the secondorder truncated expansion in the neighbourhood of $x=0$ in the isothermal centre regime implies:

$$
\mathrm{Z} \cong \frac{\mathrm{iu}\left(\mathrm{A}+\frac{\theta}{2}-\frac{3}{2}\right)}{(1+\mathrm{iu})\left(\mathrm{B}-\frac{\theta}{2}+\frac{3}{2}\right)}
$$

But, in the adiabatic centre regime, the approximation gives:

$$
\mathrm{Z} \cong \frac{\mathrm{iu}(\mathrm{A}-3)}{(1+\mathrm{iu})(\mathrm{B}+3)}
$$

In the isothermal feeding regime, the specific value of $\theta$, around which intriguing changes of response factor curve profiles are observed, can be deduced from expression (24) by cancelling the denominator, that is, by taking $\theta_{d}=2 B+3(=203$ for $B=100)$. These results confirm the important role played by the thermodynamic coefficient $B=3 \mu / \lambda$ by making, in correlation with $\theta$, high and nonlinear triggering of instability a possibility. A similar case of correlation was sufficiently highlighted in the analysis of the mean droplet pure conduction model [16] where $\theta_{d}$ has also been evaluated $\left(\theta_{d}=B\right.$ for $\left.B=100\right)$. Moreover, depending on the feeding regime, expressions of the complex transfer function $Z$ in Eq. (24) and (25) may serve to furnish estimations of peak values of response factor curves by means of their real parts. Recent studies as that of Hsiao et al. (2011), of Lafon et al. (2014) and of Sirignano (2015) $[3,5,30]$ show that the rapid variations of fluid thermodynamic properties near the critical mixing point are the major factor contributing to an abrupt change observed in droplets response. In fact, effects of fluid thermodynamic properties are rather connected to 
critical and supercritical vaporization processes through recent publications on this subject. But, the present study of the mean spherical droplet show that, an abrupt variation in the evaporating mass frequency response can occur even in a subcritical vaporization of a specifically preheated fuel. These results may be particularly beneficial for instability control in preheated and/or premixed fuel combustion systems.

\section{Conclusions}

Combined effects of thermal conduction and convection on frequency response of a vaporizing spray to pressure oscillations were studied. As in the classical Heidmann configuration, the mean spherical droplet allowed us to evaluate the mass transfer response to pressure oscillations. Two thermal forcing types were considered: constant temperature (isothermal injection regime) or zero temperature gradient (adiabatic injection regime). Approximate analytical solutions were derived for mass response factor whereby differences and similarities between the two forcing regimes were determined. Considering both types of the injection regime, comparisons were also made with the pure conduction model of the mean spherical droplet.

Important differences as well as similarities were observed between the cases analyzed. We have found that whenever the response factor has positive values pointing to instability, the maximum value is always reached at a particular reduced frequency. This particular frequency matches the period of the ambient pressure harmonic disturbances with the residence time of the mean spherical droplet. In the adiabatic feeding regime, assumed with or without thermal convection effect, the response factor peak values are shown to be almost constant relatively to the variation of the exchange coefficient. But, in the isothermal feeding case, response factor peak values vary abruptly by tending first to positive infinity when the exchange coefficient approaches a specific value while remaining lower than it, and then, to 
negative infinity once the exchange coefficient gets superior to this value. The latter is shown to be closely correlated with a certain thermodynamic coefficient that relates to fuel physical properties. These trends can be readily shown by using a phase-angle representation as mainly practiced in most time-lag models. Apart from these main differences, we saw that, for the exchange coefficient tending to zero, response factor curves are quite similar in both adiabatic and isothermal feeding regimes depending on the taking into account or not of the thermal convection effect.

This study has shown that, with or without taking into account the radial thermal convection effect in isothermal injection cases, particular correlations of the exchange coefficient with parameters depending on fuel physical properties may be a plausible cause for high frequency response occurrence in combustion chambers. Theoretical and experimental studies are still needed to clarify these issues, since the adiabatic and isothermal feeding regimes here considered are the extreme cases of a more generalized injection regime that combines the two thermal forcing types. However, the present linear analysis has the advantage to provide dimensionless parameters that may be used to characterize and examine the dynamic behaviour of the vaporization process for any fuel. The physical results obtained may also help to interpret or predict the occurrence of strong perturbations in vaporization and combustion processes. 


\section{References}

[1] Y. Huang, V. Yang, Dynamics and stability of lean-premixed swirl-stabilized combustion, Prog. Energ. Combust. Sci. 35 (2009) 293-364.

[2] S.M. Candel, Combustion Instabilities Coupled by Pressure Waves and Their Active Control, Proc. Combust. Inst. 24 (1992) 1277-1296.

[3] G.C. Hsiao, H. Meng, V. Yang, Pressure-coupled vaporization response of n-pentane fuel droplet at subcritical and supercritical conditions, Proc. Combust. Inst. 33 (2011) 1997-2003. [4] J.-P. Delplanque, W. A. Sirignano, Transcritical liquid oxygen droplet vaporization: effect on rocket combustion instability, Atomization Spray. 4 (1996) 325-349.

[5] P. Lafon, H. Meng, V. Yang, M. Habiballah, Pressure-coupled responses of lox droplet vaporization and combustion in high-pressure hydrogen environments, Combust. Sci. Technol. 186 (2014) 1191-1208.

[6] D.T. Harrje, F.H. Reardon, Liquid propellant rocket combustion instability, Technical Report No. SP-194, NASA, Washington D.C., USA, 1972.

[7] W. A. Sirignano, J.-P. Delplanque, C.H. Chiang, R. Bhatia, Liquid-propellant droplet vaporization: a rate controlling process for combustion instability, in: V. Yang, W. E. Anderson (Eds.), Liquid rocket engine combustion instability, Progress in Astronautics and Aeronautics, AIAA Inc., Reston, 1994, p. 307-343.

[8] M. de la Cruz Garc'1a, E. Mastorakos, A.P. Dowling, Investigations on the self-excited oscillations in a kerosene spray flame, Combust. Flame 156 (2009) 175-186.

[9] A. Duvvur, C.H. Chiang, W.A. Sirignano, Oscillatory fuel droplet vaporization: driving mechanism for combustion instability, J. Propul. Power 12 (1996) 358-365.

[10] A. Y. Tong, W. A. Sirignano, Oscillatory vaporization of fuel droplets in an unstable combustor, J. Propul. Power 5 (1989) 257-261.

[11] M. DiCicco, J. Buckmaster, Acoustic instabilities driven by slip between a condensed phase and the gas-phase in combustion systems, $32^{\text {nd }}$ AIAA Aerospace Sciences Meeting and Exhibit (1994), paper AIAA-94-0103.

[12] I. Dubois, M. Habiballah, R. Lecourt, Numerical analysis of liquid rocket engine combustion instability, 33 ${ }^{\text {rd }}$ AIAA Aerospace Sciences Meeting and Exhibit (1995), paper AIAA-95-0607.

[13] M.F. Heidmann, P.R. Wieber, Analysis of n-heptane vaporization in unstable combustor with traveling transverse oscillations, Report No. TN D-3424, NASA, Washington D.C., USA, 1966. 
[14] M.F. Heidmann, P.R. Wieber, Analysis of frequency response characteristics of propellant vaporization, Report No. TN D-3749, NASA, Washington D.C., USA, 1966.

[15] R. Prud'homme, M. Habiballah, L. Matuszewski, Y. Mauriot, A. Nicole, Theoretical analysis of dynamic response of a vaporizing droplet to a acoustic oscillation, J. Propul. Power 26 (2010) 74-83.

[16] K. Anani, R. Prud'homme, Theoretical analysis of thermal conduction effect on frequency response of a perturbed vaporizing spherical droplet, Flow Turbulence Combust. 98 (2017) 503-522.

[17] S.Y. Slavyanov, W. Lay, Special Functions, A Unified Theory Based on Singularities. Oxford Mathematical Monographs, 2000.

[18] L. Rayleigh , Theory of Sound (two volumes), Dover Publications, New York, 1877 . Re-issued 1945.

[19] S. A. Basha, K. R. Gopal, In-cylinder fluid flow, turbulence and spray models-A review, Renew. Sust. Energ. Rev. 13 (2009) 1620-1627.

[20] R.S. Miller, K. Harstad, J. Bellan, Evaluation of equilibrium and non-equilibrium evaporation models for many droplet gas-liquid flow simulations, International Journal of Multiphase Flow 24 (1998) 1025-1055.

[21] B. Abramzon, W. A. Sirignano, Droplet vaporization model for spray combustion calculations, Int. J. Heat Mass Transfer 32 (1989) 1605-1618.

[22] S.S. Sazhin, T. Kristyadi, W.A. Abdelghaffar, M.R. Heikal, Models for fuel droplet heating and evaporation: Comparative analysis, Fuel 85 (2006) 1613-1630.

[23] O. M. I. Nwafor, Effect of varying fuel inlet temperature on the performance of vegetable oil in a diesel engine under part-load conditions, International Journal of Ambient Energy 20 (1999) 205-210.

[24] S. Bari, T.H. Lim, C.W. Yu, Effects of preheating of crude palm oil (CPO) on injection system, performance and emission of a diesel engine, Renewable Energy 27 (2002) 339-351

[25] L. Yuan, C. Shen, Z. Xinqiao, Dynamic response of vaporizing droplet to pressure oscillation, Heat Mass Transfer 52 (2017) 711-723.

[26] G. Borghesi, E. Mastorakos, R. S. Cant, Complex chemistry DNS of n-heptane spray autoignition at high pressure and intermediate temperature conditions, Combust. Flame 160 (2013) 1254-1275.

[27] S. S. Sazhin, Advanced models of fuel droplet heating and evaporation, Prog, Energ. Combust. Sci. 32 (2006) 162-214. 
[28] R. Prud'homme, Flows of reactive fluids. Book Series: Fluid Mechanics and Its applications, Springer, 2010.

[29] K. Anani, R. Prud'homme, S.A. d'Almeida, K.S. Assiamoua, Effect of thermal convection on frequency response of a perturbed vaporizing pastille-shaped droplet, Mec. Ind. 12 (2011) 300-313.

[30] W. A. Sirignano, Driving mechanisms for combustion instability, Combust. Sci. Technol. 187 (2015) 162-205. 